\title{
INFANTS' SENSITIVITY TO EMOTIONAL ANIMAL VOCALIZATION AND THE EVOLUTION OF VOCAL COMMUNICATION
}

\author{
NAOTO YAMANE ${ }^{* 1}$, MIHOKO HASEGAWA ${ }^{1}$, AI KANATO $^{1,2}$, NAOKO KIJIMA ${ }^{1}$, \\ KAZUO OKANOYA ${ }^{3,}$ and REIKO MAZUKA ${ }^{1,4}$ \\ *Corresponding Author: yamanen@brain.riken.jp \\ ${ }^{1}$ Laboratory for language development, RIKEN BSI, Saitama, Japan \\ ${ }^{2}$ Waseda University, Saitama, Japan \\ ${ }^{3}$ University of Tokyo, Tokyo, Japan \\ ${ }^{4}$ Department of Psychology and Neuroscience, Duke University, Durham NC, \\ USA
}

For many species, transmitting emotional signals is a fundamental function of vocal communication. In pre-verbal human infants, emotions conveyed by the speech signal are perhaps the first meanings that they are able to detect (Fernald, 1993). Indeed, human newborns were shown to present an increase in eye opening responses following the presentation of happy mother's voice as compared to the other emotions (Maestropieri \& Turkewitz, 1999). By 5 months, human infants can respond appropriately to emotions conveyed by speech prosody (Fernald, 1993). Although the characteristics of vocalization that animal produce are often species specific, it appears that there is a general relationship between the physical structure of sounds and the motivation underlying their use (Morton, 1977). If different species share the ability to produce common emotional vocal signals, they may also share a common ability to detect such cues. To date, however, no studies have empirically examined whether humans or other species possess such an ability. In the present study, we examined whether human infants have sensitivities in emotional vocalizations of other species. In each of the 3 experiments, 5- and 9-month-old Japanese infants (20 in each age group) were tested using the Head-Turn Preference paradigm (HPP). The vocalizations of a naked mole rat (Exp. 1), a song sparrow (Exp. 2) and a swamp sparrow (Exp. 3) were chosen and were 
produced in either a positive (pleasure, contact) or a negative (distress, alarm) context. Infants' average listening times to the positive and the negative contexts were measured for each type of vocalizations. In Figure 1, the First Look listening time is the average listening time when the infant first oriented to the stimuli, and the Total Look listening time is the average of the total time during which infants oriented to the stimuli. For the vocalizations of the naked mole rat, infants in both age groups showed longer listening time to the negative vocalizations than the positive one both in the First Look $[\mathrm{F}(1,38)=120.56, \mathrm{p}$ $<.01]$ and the Total Look $[\mathrm{F}(1,38)=161.37, \mathrm{p}<.01]$. For the song sparrow vocalizations, 5- and 9-month-olds listened longer to the positive vocalizations than the negative ones [First Look, $\mathrm{F}(1,38)=24.7$, $\mathrm{p}<.01$; Total Look, $\mathrm{F}(1,38)$ $=30.5, \mathrm{p}<.01]$. For the swamp sparrow vocalizations, 5 -month-olds but not 9month-olds listened longer to the negative ones in the First Look $[\mathrm{F}(1,19)=$ $2.39, \mathrm{P}<.05]$, while no difference for the Total $\operatorname{Look}[\mathrm{F}(1,38)=2.26$, n.s. were found. These results could not be explained only by specific acoustic cues, such as pitch, loudness and duration. Hence, the results of our experiments clearly demonstrate that human infants possess an ability to detect some kinds of emotional vocal signals in other species. This indicates that human and nonhuman species may indeed possess a shared ability not only to produce common emotional vocal signals but also to perceive such cues.

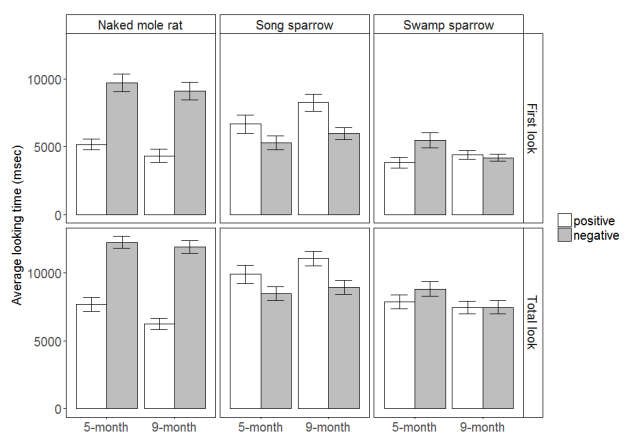

Figure 1. Mean looking times (with standard error bars) of First Look (upper part) and Total Look (lower part) for the positive (white bars) and negative (black bars) vocalizations produced by Naked mole rat (left), Song sparrow (center) and Swamp sparrow (right).

\section{Acknowledgements}

This study was supported in part by Grant-in-Aid for Young Scientists (B) (JP16K16629) to NY, Scientific Research S (16H06319) and Scientific Research on Innovative Areas \#4903 (Co-creative Language Evolution) 


\section{References}

Fernald, A. (1993). Approval and disapproval: Infant responsiveness to vocal affect in familiar and unfamiliar languages. Child development, 64(3), 657674.

Mastropieri, D., \& Turkewitz, G. (1999). Prenatal experience and neonatal responsiveness to vocal expressions of emotion. Developmental psychobiology, 35(3), 204-214.

Morton, E. S. (1977). On the occurrence and significance of motivationstructural rules in some bird and mammal sounds. The American Naturalist, 111(981), 855-869. 